\title{
What Is the Best Strategy for Preclinical Testing of Botanicals? A Critical Perspective
}

Authors

Affiliations
Veronika Butterweck ${ }^{1}$, Adolf Nahrstedt ${ }^{2}$

${ }^{1}$ Department of Pharmaceutics, College of Pharmacy, University of Florida, Gainesville, FL, USA

${ }^{2}$ Institute of Pharmaceutical Biology and Phytochemistry, Westf. Wilhelms-University, Münster, Germany
Key words

- preclinical testing

- ethnopharmacology

- ethnopharmacy

- botanicals

in vivo

- in vitro

- dosing

- application

received January 7, 2012

revised February 29, 2012

accepted March 12, 2012

Bibliography

Dol http://dx.doi.org/

10.1055/s-0031-1298434

Published online April 12, 2012

Planta Med 2012; 78: 747-754

(c) Georg Thieme Verlag KG

Stuttgart · New York .

ISSN 0032-0943

\section{Correspondence}

Prof. Dr. Veronika Butterweck

Department of Pharmaceutics

College of Pharmacy

University of Florida

Gainsville, Florida 32610

United States

Present address: Institute for

Pharma Technology

School of Life Sciences

University of Applied Sciences

Northwestern Switzerland

Gründenstrasse 40

4132 Muttenz

Switzerland

Phone: + 41614674689

veronika.butterweck@fhnw.ch

\section{Correspondence}

\section{Prof. Dr. Adolf Nahrstedt}

Institute of Pharmaceutical

Biology and Phytochemistry

Westfälische Wilhelms-

Universität

Hittorfstrasse 56

48149 Münster

Phone: + 49251262599

anahrstedt@uni-muenster.de

\section{Abstract}

$\nabla$

The development of a new drug is generally marked by a number of preclinical investigations in a sequential order with regard to contents and logic. However, ethnopharmacology often uses the "reverse pharmacology" approach, which is based on anecdotal therapeutic effects of plants in ancient texts or based on the empirical knowledge of traditional healers. While this approach could successfully lead to new therapeutic applications by using sophisticated techniques and appropriate bioassays in a logical order, unfortunately there is an exponentially increasing number of reports of pharmacological effects of botanical extracts with insignificant bioactivities obtained in often irrelevant in vitro bioassays. The interpretation based on in vitro data can only be misleading since the pharmacokinetic properties of a compound are ignored, unacceptable high dosages of extracts are tested, or metabolism to

\section{Introduction \\ $\nabla$}

It is generally estimated that there are approximately between 250000 and 500000 species of higher plants [1]. Approximately 10000 of the world's plants have documented medicinal use, and more than $90 \%$ of current therapeutic classes derive from a natural product prototype $[1,2]$. The discovery of such prototypes has led to significant changes in the practice of medicine; just consider the impact of Taxol ${ }^{\circledR}$, which was initially discovered from Taxus brevifolia, on cancer therapy. However, some of these prototype discoveries have been made accidentally, often following the "trial and error" principle. For example, the identification of penicillin in Penicillium mold by Alexander Fleming in 1928 is one of the bestknown stories of medical discovery, not only because of its accidental nature, but also because inactive metabolites is not considered. Further, many natural products are prodrugs that need to be metabolized in vivo by the intestinal microflora or by mammalian phase I/II metabolism. Frequently, attempts are made to master poor pharmacokinetics by administering the extract intraperitoneally or intravenously, clearly moving away from the traditional oral application. In this review article, it is proposed that preclinical testing strategies of botanicals should start with the in vivo examination of extracts in relevant animal models to substantiate the ethnopharmacological/ethnopharmaceutical use, followed by bioguided fractionation processes using an adequate in vitro model, further followed by pharmacokinetic studies and final in vivo testing of isolated compounds. With our article we would like to encourage authors, reviewers and editors to implement this strategy for the design of experiments and for the reviewing and editing process of manuscripts.

penicillin has remained one of the most important and useful drugs, and its discovery triggered invaluable research into a range of other invaluable antibiotic drugs. Other important drugs that were discovered by chance and are of plant origin are quinine, cocaine, digoxin, and aspirin (with salicylic acid as the model), just to mention a few. Microbiologist and historian Alexander Kohn stated once: "Nearly all great discoveries in chemotherapy have been made as a result of a false hypothesis or due to a so-called chance observation" [3]. However, although accidents play an important role in drug discovery, the ability of the scientist is required to find importance in the observations and to recognize their significances. In this context, Pasteur formulated in 1854, "in the field of observation, chance only favors the prepared mind". 
Nowadays, drug targets and disease mechanisms twisted empiric drug discovery to rational drug design programs. Thus, with all the advances in the fields of medicinal chemistry and bioassay technology, the odds for the discovery of a new prototype drug from natural sources based on sound ethnopharmacological principles should be significantly better than the odds for the discovery of a new drug from a synthetic high-throughput drug discovery program. Especially since there is a productivity crisis in drug discovery and development departments in pharmaceutical companies, with R\&D presently being at an all time low level with only 21 new launches in 2010, compared to 26 during 2009 [4]. The exhaustion of drug-pipelines in the near future presents a global risk for the public health and safety and clearly raises questions and concerns regarding new sources of lead structures for drug discovery.

Considering the fact that only a fraction of the world's plants has been investigated, the question arises why plant-based drug discovery is so disgracefully inefficient then? Why did large scale and expensive botanical research projects that have been assigned to reputable groups and funded by governmental agencies or industrial companies embarrassingly fail? Why does the general belief exist in the scientific community that botanical research should be avoided for the sake of one's professional reputation? Why do the same people think that good botanical research is impossible to do and just a waste of time? Where is this attitudinal barrier coming from that discourages botanical research? Interestingly, the same questions have been raised almost 30 years ago by Malone [5], but we still haven't resolved fundamental issues associated with ethnopharmacological/ethnopharmaceutical research. So, where did we fail and what can we do to fix this dilemma?

Surprisingly, Malone [5] already presented a sound strategy in the early ' 80 s for research in ethnopharmaceuticals with the aim to discover new prototype drugs. According to his article, natural product research is best conducted in a systematic fashion concentrating on certain critical questions that should be answered in a sequential fashion by appropriately trained experienced professionals. Further, one must not try to do two or three steps or all steps simultaneously [5].

The rational drug development process passes through several stages which should follow a logical order. However, when looking at screening programs based on ethnopharmacology, it appears that experiments are arranged in an illegitimate and random order and are often far away from being multidisciplinary. For example, is it intelligent to start a botanical extract screening program with in vitro assays that are designed to unravel the mechanism of action when it turns out later in the program that the extract has no activity when tested in an in vivo assay? What relevance do data from fancy molecular biology assays have when we do not have any information about the pharmacokinetics of a particular natural product? Is it good science when mechanisms of action of extracts are hypothesized based on pharmacological assays without knowing anything about the phytochemical composition of the extract? How meaningful are the data when unrealistically high doses of extracts/compounds are necessary to achieve a pharmacological effect? And, last but not least, why do these papers get published?

Almost thirty years after Malone's publication, this article is now a new attempt to discuss these problems and to present an updated strategy for preclinical testing of botanicals.

\section{Program Differences and the Dilemma of Insignificant Pharmacological Activities $\nabla$}

In rural areas where civilization has almost no influence, natives must rely on effective plants to treat diseases since synthetic medications are not available. Thus, no person willingly would take an ineffective drug or a plant that is acutely toxic. The basis for selecting plants with beneficial effects and low toxicity relies on careful observation, description, and experimental investigation of indigenous drugs and their biological activities [5]. On the other hand, the development of a new medicinal product is generally marked by a number of preclinical and clinical investigations that follow a sequential order with regard to contents and logic. As every new active substance is a separate entity, investigations and testing strategies must be designed individually [6]. Compared to this, ethnopharmacology/ethnopharmacy uses the "reverse pharmacology approach" in which the candidate travels a reverse path from "clinics to laboratory" rather than the classical "laboratory to clinics". The concept of "reverse pharmacology" was created in India to develop pharmaceuticals from Ayurvedic medicines [7]. Since substantial experience of human use exists, the chances that a remedy will be effective and safe and that precautions will be known are relatively high. The concept is inspiring and can offer a smart strategy not only for the identification of new lead compounds but also for the development of rational phytotherapeutics. Nevertheless, some basic considerations are important. As Heinrich pointed out recently [8], one of the major challenges in ethnopharmacology/ethnopharmacy is to accurately perform a scientific study with a plant extract since it is a multicomponent mixture of active, partially active, and inactive compounds. Thus, accurately performed pharmacological experiments rely on critical approaches and well elaborated experimental designs. The experiments not only have to be executed professionally, even more important is the careful examination of the results in order to avoid an overinterpretation of the data. The dilemma today is, however, that there is a trend to attribute a pharmacological effect to almost every plant extract [9]. This leads to serious misinterpretations, especially when data were generated based on in vitro assays. But does anybody care? Unfortunately this is not the case. Clear standards and validated assays are pivotal but are often ignored since they would put results that sound too good to be true into question. If every health claim of a botanical extract would be true, we already would have the cure for cancer, diabetes, cardiovascular and other severe diseases. We all know that this is not the case. In his article, "How scientific is the Science in Ethnopharmacology?" Gertsch [9] gets to the heart of the problem by saying that "nonsense in bioassays represents an increasing phenomenon in botanical research".

From the perspective of a journal editor, it should be alarming that authors submit their manuscript to a journal without performing a critical assessment of their working hypothesis. Instead, researchers follow the path of the least resistance and jump to new projects, thus, generating even more background noise data [9]. It is even more striking that manuscripts reporting insignificant pharmacological activities of extracts and isolated compounds pass the editors and reviewers of a journal and are made available for a broader scientific community. As a consequence, the reputation of our discipline is severely damaged. We think that the scientific community has the biggest responsibility to influence how data are reported and how scientific pharmacological research with botanicals should be. In fact, journal editors have the greatest responsibility and power to guide and shape 
the scientific content of a manuscript as already proposed by Kroll and Oberlies [10].

So, what are common experimental problems and potential pitfalls in pharmacological assays that are not recognized by editors and reviewers? And, more importantly, are there ways to differentiate between facts and artifacts? The following paragraphs will highlight some of the most frequently made mistakes in pharmacological assays.

\section{Fact or Artifact?}

$\nabla$

Pitfalls in the selection of an appropriate model and sufficient sample size

Pharmacologists have the job to find new therapeutic drugs by using appropriate models. These pharmacological models have to be relevant, which means that they should be able to predict the intended therapeutic indication [11]. A pharmacological model can be considered relevant when the effects obtained correlate with the results observed in human therapy. The reference book "Drug Discovery and Evaluation" contains a comprehensive selection of the most frequently used assays for reliably detecting the pharmacological effects of potential drugs [11]. Effects covered include cardiovascular, analgesic, endocrine, psychotropic respiratory, renal, and immunomodulatory activities. Each of the more than 1000 assays comprises a detailed protocol outlining the purpose and rationale of the method, a critical assessment of the results, and their pharmacological and clinical relevance. This book is highly recommended to scientists performing pharmacological assays.

One of the basic recommendations inside the book is the selection of an appropriate model that has to be sensitive in a dose-dependent fashion to a standard reference compound. A standard reference compound (= positive control) is a substance that is known to possess the desired therapeutic property [11]. Furthermore, the potency of the positive control in the model should be comparable to their relative potency in clinical use. Unfortunately, if we take a closer look at pharmacological assays performed with herbal extracts, we notice that this basic rule is often ignored. It would be too laborious to search the entire literature for papers that describe pharmacological effects of plant extracts where positive controls were not applied. In only very few cases positive controls are not available, for example if assays are applied to indications for which no effective drug is known. In such a case, the exclusion of a reference control from a study should be justified. In general, for standard pharmacological assays that are used to investigate botanicals (in the treatment of cancer, diabetes, depression, anxiety, Alzheimer's, obesity, inflammation, etc.), internationally accepted reference compounds do exist. If no positive control was used in a standard assay, it is impossible to interpret the resulting data with regard to their scientific content. A positive development is that journals in the natural products field such as Planta Medica, Journal of Ethnopharmacology, Journal of Natural Products, Phytomedicine, and Phytotherapy Research have developed guidelines that will not consider manuscripts where pharmacological effects of extracts or isolated compounds are reported without comparison with an internationally accepted positive control.

A further issue that needs to be addressed is the correct use of statistical tools. The first question always should be what sample size is required for an experiment to determine the nature of a dose response to a drug. According to the ICH guideline for safety pharmacology studies for human use, the size of the groups should be sufficient to allow meaningful scientific interpretation of the data generated [12]. Thus, the number of animals or isolated preparations should be adequate to demonstrate or rule out the presence of a biologically significant effect of the test substance. In other words, good experimental design is important in order to answer the research question of interest in a way that is free of bias, can be generalized to the desired or targeted population, and is of sufficient size to properly answer the question [11]. This includes such things as determining the measurements to make, timing, dosing frequency, and route, the species to use, etc. It also includes identifying the relevant statistical analyses, determining appropriate sample sizes and a randomization scheme.

Before carrying out an assay, appropriate statistical methods, including sample size calculations and software for analyzing the data from these experiments, should be determined. There are several statistical methods available to analyze any given experiment/data set, and the choice of these methods and the way a certain class of methods is implemented can significantly impact the conclusions from the experiment. For example, there are certain statistical considerations one should take into account when using the analysis of variance (ANOVA) method, including the distribution of the data, equality of variances, baseline variables, methods for comparing different groups, etc. Also, Student's ttest might often seem appropriate for several types of experiments, but upon careful examination of the study design (e.g., when more than two groups are compared), the t-test might turn out to be less appropriate than some of the other statistical analysis methods. If the study design is changed at any time during a series of experiments, appropriate analysis methods and implementation strategy should once again be examined in light of these changes. General information about how to design proper pharmacological assays can be found under http://assay.nih.gov/ assay/index.php/Table_of_Contents. However, these basic rules are often ignored in ethnopharmaceutical research, and unfortunately, it is quite common that authors use for example, a sample size of $n=4$ per group, comparing, e.g., 8 different experimental groups with each other, trying to do a statistical data analysis using Student's t-test. Such data are not scientifically meaningful and can be disregarded.

A further concern is that very often different routes of administration for the positive control and the extract/natural compound are compared. For example, in a recent paper, the authors Adeyemi et al. [13] investigated the anticonvulsant, anxiolytic, and sedative effects of an aqueous root extract of Securidaca longepedunculata, a plant that is used in traditional African medicine to treat epilepsy. In all experiments, the extract was administered orally while the positive control diazepam was injected intraperitoneally. The vehicle control solution was given via different routes, depending on the experiment. From a pure statistical standpoint, it is possible to compare these groups. Statistics can be correctly applied to a lot of nonsense; however, the results won't make much sense. One should only compare different compounds/extracts administered by the same route (unless one wants to evaluate differences due to the routes). The route of administration in this case is an additional factor that needs to be involved in the statistical analysis, since it could cause differences in the outcome. At least, there should be a good reason given why authors used a different route of administration for the positive control and the test substance, and this would need to be discussed in the paper but the results are not very meaningful then. If the ex- 
tract and the control were given orally, it would be the best to give diazepam orally as well.

The pharmacokinetic consequences of different routes of administration will be discussed in detail in one of the following paragraphs.

\section{Pitfalls in the use of in vitro assays}

In vitro bioassays are commonly used in botanical research to screen new extracts and isolated compounds and to evaluate their mechanism of action. These assays are inexpensive and relatively easy to perform. Typically, cells are grown at the bottom of a culture well, and the test material is applied in several doses to measure a dose-response relationship. However, performing in vitro bioassays with botanical extracts is a permanent challenge since they are multicomponent mixtures. Traditionally, in vitro assays have been designed for testing soluble molecules. Yet, many herbal extracts are not water soluble; therefore, a solvent that is not toxic to cells has to be selected and used as a control. Very often DMSO is used as a solvent for extracts. The disadvantage is that DMSO might also have an effect on the cells [14]. In fact, all cells will be affected by concentrations of DMSO above $0.1 \%$. To take this into consideration, one should always have a control setting where the cells are treated with everything but the herbal extract, so in this case just with DMSO. Further, dissolving the extract in DMSO might solubilize compounds that under more moderate conditions never would have been dissolved, so that in the assay, effects of compounds might be measured that have no relevance for the therapeutic effect after the experiment was tested in in vivo assays. From a solubility aspect, testing plant extracts in in vitro assays is problematic since they are considered as solid objects (or "fibers") that do not behave in the same way as soluble molecules; therefore, it will be difficult to define an appropriate expression for the dose [15]. Larger nanoparticles or microparticles in extracts generally sediment rapidly and come into contact with the cells, thus inducing unwanted oxidative stress or even toxic effects. A possible solution to address this drawback could be to centrifuge the extract before testing and to apply only the clear supernatant to the in vitro assay. However, in this case it will be important to perform a phytochemical analysis and to determine the composition of the supernatant. In vitro testing can continue if there are no significant changes in the phytochemical composition of the extract after centrifugation. If the results are affected by particles in the extract, then this issue needs to be addressed and analytical or computational assessment of the cellular dose in vitro will be required [15]. Unfortunately, in vitro bioassays are often carried out carelessly, and these procedures are not considered, thus misleading data are generated.

Another issue that needs to be pointed out is that very often outrageously high concentrations of extracts are used in in vitro assays to obtain a dose-response effect. This topic has been nicely addressed by Gertsch [9] who provided several examples in which claims of activities at absurd concentrations are extrapolated to the human situation despite the lack of a clear scientific basis for such assumptions. To provide an additional example, Subramanian et al. [16] investigated an ethanolic extract of Andrographis paniculata for $\alpha$-glucosidase inhibitory activity and found an "appreciable" inhibitory effect at an $\mathrm{IC}_{50}$ of $17 \mathrm{mg} / \mathrm{mL}$ (!) and a "weak" inhibitory effect at an $\mathrm{IC}_{50}$ of $50 \mathrm{mg} / \mathrm{mL}$ (!). The authors conclude that $\alpha$-glucosidase inhibition could be a possible mechanism of action of $A$. paniculata which therefore could be considered as a potential candidate for the management of type 2 diabetes. The potency of this effect is meaningless when we consider that the $\alpha$-glucosidase inhibitor acarbose shows $\mathrm{IC}_{50}$ values of $0.3 \mu \mathrm{g} / \mathrm{mL}$ [17]. A further example is the proposed anti-gout activity of Tahitian Noni juice (Morinda citrifolia L.) as suggested by Palu et al. [18]. Concentrations of 1, 5, and $10 \mathrm{mg} /$ $\mathrm{mL}$ of the juice inhibited xanthine oxidase by $11 \%, 113 \%$, and $148 \%$, respectively, with an $\mathrm{IC}_{50}$ value of $3.8 \mathrm{mg} / \mathrm{mL}$ (!) compared to an $\mathrm{IC}_{50}$ of $2.4 \mu \mathrm{g} / \mathrm{mL}$ of the standard allopurinol. Although both examples lack any scientific merit and the data are artificial, they present good examples for the sort of background noise data that is being generated in ethnopharmacology/ethnopharmacy. It is further remarkable that papers like the two presented examples not only got published but most likely will be uncritically cited by other authors. However, the general problem is that clear standards for in vitro testing of extracts are missing. A clear definition about what can be considered as active is needed to stop wasting time and research funds on plants that only have weak activities [19]. An approach in this direction has been taken by Cos et al. [20] on testing microbial activity in plants. The review provides very helpful recommendations for defining bioassays for antibacterial, antifungal, and antiparasitic activity, and some of the suggestions can be used as a general guideline for in vitro testing, such as stringent endpoint criteria with $\mathrm{IC}_{50}$ values below $100 \mu \mathrm{g} / \mathrm{mL}$ for extracts and below $25 \mu \mathrm{M}$ for pure compounds [20].

However, even if an extract or pure compound shows a hit in an in vitro bioassay, this is not a guarantee that they will be active in a matching in vivo assay. We recently examined the xanthine oxidase (XO) inhibitory activity of an artichoke leaf extract (ALE) and some of its main compounds in vitro and then further tested potentially active substances of this extract for possible hypouricemic effects using an in vivo rat model [21]. The in vitro study showed that ALE inhibited XO with only minimal inhibitory action $(<5 \%)$ at $100 \mu \mathrm{g} / \mathrm{mL}$. However, when selected compounds were tested, the aglycone luteolin potently inhibited XO with an $\mathrm{IC}_{50}$ value of $1.49 \mu \mathrm{M}$ compared to the reference compound allopurinol with an $\mathrm{IC}_{50}$ of $3.85 \mu \mathrm{M}$. Although detected as a promising hit in vitro, oral administration of luteolin did not produce any observable hypouricemic effects after acute oral treatment in potassium oxonate-treated rats [21]. In our particular case, the in vitro properties of luteolin could not be correlated with its in vivo performance due to the low bioavailability of luteolin [22]. In general, cells and tissues are physiologically connected, and this interplay may be crucial in the evolution of some disorders. Thus, many of the hits generated by traditional in vitro screening of extracts or natural products turn out to be invalid once tested in animals, resulting in a waste of funds and efforts. Absorption, solubility, distribution, metabolic stability, and toxicological problems in later animal studies present numerous difficulties that turn to a dead-end for most hits. These aspects have to be considered before researchers get carried away with the interpretation of their data from in vitro assays.

The challenge for the investigator always is to correlate in vitro data with in vivo findings, bearing in mind the old saying "In vitro simplicitas, in vivo veritas" (in vitro simplicity, in vivo the truth) [11]. The effects found in tissue cultures are quite often not typical for an intact organism. Unfortunately, many plant projects never pass the in vitro level. Instead, findings from in vitro studies are directly up-scaled to the human situation, and we talk about efficacy in humans but mean effects observed in a cell-based assay [9]. This clearly needs to be improved and should also be implemented in all journal instructions for authors. 


\section{Pitfalls in the dose translation from animal to} human studies

Biomedical research needs in vivo animal experiments which provide a framework for human clinical trials. It is therefore essential in the drug discovery process to accurately translate the drug dosage from animal to human studies. Unfortunately, there is a general misunderstanding and confusion about how to appropriately translate a drug dose from animal to human studies. Very often the animal dose is extrapolated to a human equivalent dose (HED) by simple conversion based on body weight. ReaganShaw et al. [23] nicely showed in their article about dose translation from animal to human studies the fatal misinterpretation of data based on such an assumption. In their article, they discuss the study of Baur et al. [24], in which a dose of $22.4 \mathrm{mg} / \mathrm{kg}$ body weight of resveratrol in mice was found to protect against the diseases of aging. The media reported that a $60 \mathrm{~kg}$ human would have to consume $1344 \mathrm{mg}$ of resveratrol $(22.4 \times 60)$ per day in order to achieve health benefits. Based on the assumption that a bottle of red wine contains approximately $2 \mathrm{mg}$ of resveratrol, a person would have to drink 672 bottles of wine [23]. This presents a serious misinterpretation of the research. For a more accurate conversion of drug doses, the Food and Drug Administration [25] suggested that the extrapolation of animal to human doses $(\mathrm{mg} / \mathrm{kg})$ is correctly performed through normalization to the body surface area $\left(\mathrm{BSA}, \mathrm{mg} / \mathrm{m}^{2}\right)$. The corresponding equation can be found in the paper of Reagan-Shaw et al. [23]. Using this equation to convert the dose in a mouse to a dose based on the surface area for humans, the equivalent resveratrol dose would result in $1.82 \mathrm{mg} / \mathrm{kg}$ which would correspond to $109 \mathrm{mg}$ for a $60 \mathrm{~kg}$ person. However, this still would mean that a person has to consume 55 bottles of wine in order to see health benefits which might not be reasonable. This, on the other hand, points to another severe issue that will be addressed in the next paragraph: the route of administration and the pharmacokinetic consequences of it.

\section{Pitfalls in the route of administration and the pharmacokinetic consequences}

What is the best route of administration for extracts or natural compounds when performing animal experiments? In general, the expected clinical route of administration should be used when feasible [12]. Since almost all botanical preparations traditionally have been used in the form of infusions, decoctions, or as macerates for oral consumption, extract and isolated compounds should be used using the same route of administration. Unfortunately, there are several examples in the literature where extracts have been administered intraperitoneally or even intravenously, thus moving away from the traditional ethnomedical application. The questions that have to be asked are: Does it really matter if a plant extract/natural compound is administered orally or intraperitoneally? Will the results change?

As with other practical issues discussed in this article, some basic considerations apply before it is possible to answer this question correctly. Basic principles of how to properly choose the route of administration are comprehensively summarized by Nebendahl and Hauff [26]. The following just gives a summary of some of their recommendations.

Numerous sites of administration have been described in the literature for administering substances to rodents. The route that is most frequently used is the intraperitoneal (i.p.) injection of compounds. Basically, i.p. injections are injections into a black box, and for this reason, the procedure causes a number of con- cerns that have been pointed out critically by Svendsen in his article "Ethics and Animal Welfare Related to in vivo Pharmacology and Toxicology in Laboratory Animals" [27]. The advantage of i.p. injection is that it is very easy to perform compared to many other routes of administration. As Svendsen [27] correctly emphasizes, with i. p. injection there are absorption, tolerance, and misplacement issues to be taken into consideration. Absorption can vary considerably with respect to visceral absorption depending on where the injection is placed [28]. In case of visceral absorption the drug absorbed will subsequently enter the portal vein and may be subjected to first-pass metabolism in the liver. The visceral absorption varies in different parts of the abdominal cavity. Drug formulations might cause irritation to the parietal and visceral peritoneum and the underlying tissues when injected intraperitoneally. This is well known for formulations of barbiturates, with acetic acid [29], and many other formulations, and this also applies for extracts, especially when they are injected as suspensions. The irritation may affect the absorption rate and in addition cause peritoneal and visceral pain. Further, i.p. injections of different formulations have been used as a tool to induce peritoneal sclerosis in mice and rats [29]. All these factors add to the variation in pharmacokinetic and pharmacodynamic endpoints to be measured. In addition, the procedure has been shown to be potentially stressful to the animals as indicated by expression of Fos antigens in discrete areas of the brain of mice after i.p. injection of normal saline [30] or by elevation of the nociceptive threshold after i.p. injection of hypertonic saline as stress-induced analgesia [31]. These often neglected factors will have an impact on the results and can lead to serious misinterpretations. For example, de-Paris et al. [32] studied an alcoholic extract of Pfaffia glomerata roots in several behavioral models to screen for central nervous activities. They injected the extract in doses of 100,500 , and $1000 \mathrm{mg} / \mathrm{kg}$ using the intraperitoneal route and compared the activity in different models to oral administration using the same doses. After i. p. injection of $500 \mathrm{mg} / \mathrm{kg}$, the extract decreased open field activity and memory retention in step-down avoidance but did not have an effect in the elevated plus maze or the forced swimming test. After oral administration, no effect on memory retention was observed. The authors conclude that the ethanolic extract of Pfaffia glomerata shows central depressant effects when administered intraperitoneally while after oral administration no effects where observed [32]. As shown in $\odot$ Fig. 1 extracts in dosages up to $1000 \mathrm{mg} / \mathrm{kg}$ given in a volume of $10 \mathrm{~mL}$ (water) are most likely suspensions rather than clear solutions. The injected particles therefore will cause ir-

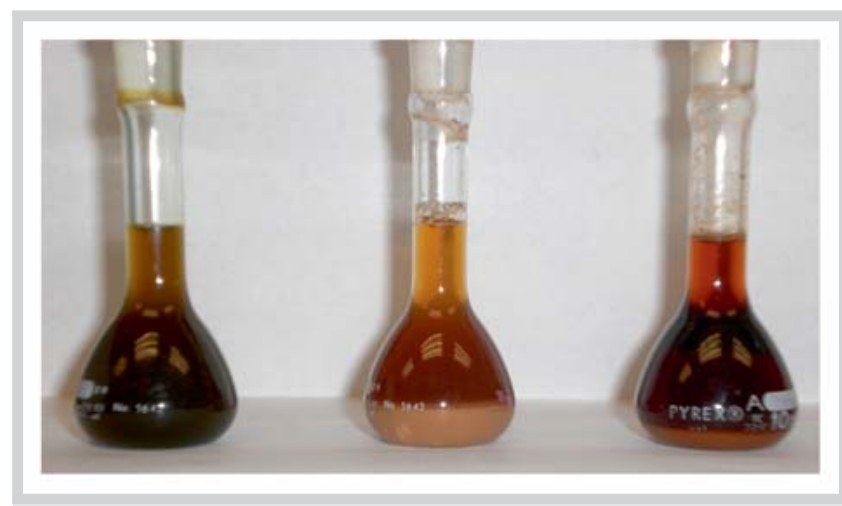

Fig. 1 Various plant extracts whose dosage of $1000 \mathrm{mg} / \mathrm{kg}$ is suspended in $10 \mathrm{~mL}$. 
ritation and peritoneal and visceral pain [27]. Thus, it is more likely that the decreased effects in the open field observed by de-Paris [32] are not due to any central depressant effects but are rather an indicator of the pain the animals were in during the test. The discussed paper represents just one example of an artifact that is often published in ethnopharmaceutical research involving laboratory animals. Unfortunately, this is not a single case, and there are a lot of such papers circulating in the literature.

So, if i.p. injection is not the method of choice, what should be the preferred route of administration? According to Nebendahl and Hauff [26], the preferred route of administration is the enteral one which involves the introduction of substances into the gastrointestinal tract via the mouth using a feeding needle. The advantage of enteral administration is the fact that it makes it possible to give comparatively large amounts of nonsterile substances or solutions. Further, a pH value as low as 3 can be tolerated for a solution whereas alkaline solutions are only poorly tolerated. In principle, absorption of substances takes place over the whole length of the digestive tract. Because of its extensive surface area and rich blood supply, the upper small intestine of rodents is the major site of absorption for all substances after oral administration. When the oral route is used, it needs to be taken into account that enzymes of the intestinal microflora can metabolize substances. These microorganisms are found under physiological conditions only in the large intestine, and the enzymatic metabolism applies only to those substances that are not absorbed in the upper intestinal tract. The disadvantage of oral administration therefore is that enzymes of microorganisms can alter substances before they are absorbed in the bowel [26]. If the metabolites are not biologically active, the administration of these substances might not be affected.

However, it has to be considered that natural products are often prodrugs. In general, a prodrug must undergo metabolic conversion before becoming an active pharmacological agent. There are several examples presented in the literature, e.g., the bioactivation of the isoflavone daidzein to the active metabolite equol or the metabolic activation of the phenolic compound salicin from willow bark by gut bacteria to salicylic acid $[33,34]$.

There is increasing evidence in the literature that flavonoids also could be potential prodrugs. Several in vivo and in vitro studies have confirmed that flavonols are metabolized by the intestinal microflora to their corresponding hydroxyphenylacetic acids [35-41]. We recently compared the anxiolytic activity of the flavonols kaempferol, quercetin, and myricetin in the elevated plus maze after oral and intraperitoneal administration to mice in a dose range of 0.1 to $2.0 \mathrm{mg} / \mathrm{kg}$ [42]. In addition, we have tested their corresponding metabolites p-hydroxyphenylacetic acid ( $\mathrm{p}$ HPAA) and 3,4-dihydroxyphenylacetic acid (DOPAC). Anxiolytic activity was detected for kaempferol and quercetin only after oral administration. No anxiolytic effects were observed when kaempferol and quercetin were given via the intraperitoneal administration route. The corresponding hydroxyphenylacetic metabolites p-HPAA and DOPAC showed anxiolytic effects after intraperitoneal application. In order to further test the hypothesis that flavonoids are possible prodrugs that require activation by intestinal bacteria, gut sterilization was performed using pretreatment with the antibiotic enrofloxacin. After antibiotic treatment, the anxiolytic effect of orally given kaempferol and quercetin disappeared, whereas it was still present for the positive control diazepam [42]. Our recent data provide the first evidence that the route of administration determines the biological activ- ity of flavonoids. The lack of an anxiolytic effect of kaempferol and quercetin after intraperitoneal injection as well as gut sterilization with enrofloxacin supports this hypothesis. In summary, the route of administration really does matter since it has a major influence on the pharmacokinetics of a compound.

Another thought that has to be considered in this regard is the fact that co-effectors (influence solubility and/or transport of active constituents) in plant extracts improve the bioavailability of active constituents as it was observed for hypericin (pharmacokinetic synergy) $[43,44]$. Such positive interactions between single compounds seem to occur more frequently in traditionally used herbal preparations than is known presently [44]. However, such interactions would not be detected using the i. p. injection route. Considering the advantages and disadvantages of oral and intraperitoneal administration, what would be the easiest method of administration? According to Nebendahl and Hauff [26], the simplest method to administer a substance is to mix it with food or drinking water. However, this method is neither very accurate nor is it practicable with substances that are insoluble or chemically unstable. Mixing compounds into food must be done carefully and accurately, especially when extracts are incorporated, since they represent multicomponent mixtures that might contain compounds that are sensitive to heat or pressure to which they are exposed during the preparation process. Thus, it will be necessary to determine release and recovery rates from food pellets in order to estimate the amount of compounds that will be taken up by the animals. Further, the daily food and water intake of the animals must be known before starting the experiment so that the amount of substance to be mixed with food or drinking water can be accurately calculated [26]. Oral administration by diet or drinking water is not suitable when the exact amount of substance intake is required since food and water wastage is very common in rodents. Therefore, it is impossible to determine the exact amount of diet or water intake of rodents without placing them into metabolic cages [26]. There are numerous examples circulating in the literature where these basic considerations where not taken into account, and the consequences of carelessly applying different routes of administration are fatal since they can lead to wrong conclusions and serious misinterpretations.

\section{Summary and Conclusion}

$\nabla$

The present article highlighted some of the major problems in ethnopharmacological/ethnopharmaceutical research. As pointed out, these limitations often have their roots in poor methodological setups and uncritical assessment of the data. This rather unscientific behavior is unfortunately facilitated by the lack of clear standards of how to perform meaningful pharmacological experiments using plant extracts and natural products. Further, the immense publication pressure fosters the malpractice of publishing premature observations without critical evaluation of the results [9]. The intention of this article therefore is to encourage authors, reviewers, and editors to stop the fabrication of unscientific hypotheses by critically and rationally planning, executing, and evaluating the experiments. Journal editors could be especially influential in requiring implementation of these state of the art experimental standards into journal guidelines. So, what could be a possible strategy for preclinical testing of botanicals? What are the recommended experimental Do's and Dont's? Based on our analysis, we think that a stepwise approach (๑ Fig. 2) is best suited for preclinical testing of botanicals. The 


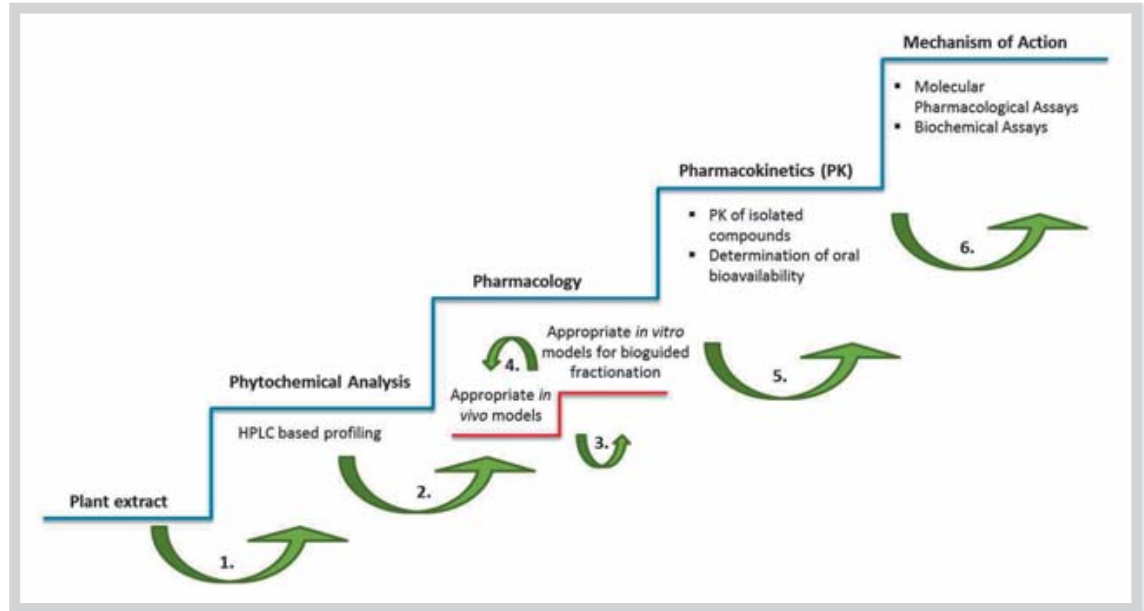

Fig. 2 Illustration of a stepwise approach for preclinical testing of botanicals based on a sequential order.



Fig. 3 Checklist with practical basic considerations when planning a pharmacological experiment.

first step should start with the phytochemical analysis of the plant extract in order to determine a possible compound pattern. In a next step, the extract should be examined in vivo in relevant animal models after oral administration in appropriate doses to substantiate the ethnopharmacological use. Once the extract was tested positively in the in vivo model, a bioguided fractionation process using an adequate in vitro screening model in relevant doses should follow; however, one should be aware that coeffectors may be present if the activity decreases or even disappears during purification [44]. These types of interactions between compounds are combined under the term "pharmacokinetic synergy"; unfortunately, they are not well investigated in herbals, particularly at the molecular level [44]. At this point, we would like to refer the readers to the recent article of Gertsch [45] entitled "Botanical Drugs, Synergy, and Network Pharmacology: Forth and Back to Intelligent Mixtures", in which the overall concept of synergy and polypharmacology concepts are discussed in greater detail, this being beyond the scope of the present article. Attention should be paid to the solvent used and the $\mathrm{IC}_{50}$ determined. A fraction that yields into a positive hit should be further investigated in the selected in vivo model until a single active compound is identified. As soon as a single active compound is detected, a pharmacokinetic profile should be determined followed by a final in vivo testing of the isolated compound. Molecular pharmacological or biochemical assays can be carried out as a very last step to evaluate the mechanism of action. These assays only provide useful data with pure isolated compounds when information regarding their in vivo activity and bioavailability are available. Nonsense data are generated when extracts are applied to (in vitro) molecular pharmacological assays or if isolated compounds are tested on a molecular level before information regarding their bioavailability is available. In addition, basic considerations on how to perform pharmacological assays need to be taken into account such as the physicochemical properties of the testing material, choice of realistic doses, adequate test models, appropriate routes of administration, and suitable statistical evaluations that will enable proper data interpretation (see checklist provided in 0 Fig. 3). We think that by implementing these state of the art experimental standards and the suggested testing strategy into guidelines for authors and instructions for reviewers and editors, the number of background noise manuscripts will drop significantly but the credibility of pharmacological research with botanicals and natural products will be restored.

\section{Conflict of Interest \\ $\nabla$}

The authors declare no conflict of interest. 


\section{References}

1 McChesney JD, Venkataraman SK, Henri JT. Plant natural products: back to the future or into extinction? Phytochemistry 2007; 68: 2015-2022

2 Newman DJ, Cragg GM, Snader KM. Natural products as sources of new drugs over the period 1981-2002. J Nat Prod 2003; 66: 1022-1037

$3 \mathrm{Kohn}$ A. Fortune or failure: missed opportunities and chance discoveries. In: Roberts RM, editor. Serendipity: accidental discoveries in science. New York: Wiley; 1989: 123-125

4 Taylor L. Pharma R\&D spend, NME launches slumped in 2010: study. Available at http://www.pharmatimes.com/article/11-06-28/Pharma_ R_D_spend_NME_launches_slumped_in_2010_study.aspx. Accessed January 2012

5 Malone $M$. The pharmacological evaluation of natural products - general and specific approaches to screening ethnopharmaceuticals. J Ethnopharmacol 1983; 8: 127-147

6 Olejniczak K, Guenzel P, Bass R. Preclinical testing strategies. Drug Inf J 2001; 35: 321-336

7 Patwardhan B, Mashelkar RA. Traditional medicine-inspired approaches to drug discovery: can Ayurveda show the way forward? Drug Discov Today 2009; 14: 804-811

8 Heinrich M. Ethnopharmacology in the 21st century - grand challenges. Front Pharmacol 2010; 1: 8

9 Gertsch J. How scientific is the science in ethnopharmacology? Historical perspectives and epistemological problems. J Ethnopharmacol 2009; 122: 177-183

10 Kroll DJ, Oberlies $\mathrm{NH}$. The impact of newly proposed dietary supplement manufacturing guidelines on patient safety and clinical trial outcomes. Focus Alternative Compl Ther 2003; 8: 302-306

11 Vogel HG, Vogel W. Drug discovery and evaluation. New York: Springer; 2002

12 ICH Harmonized Tripartite Guideline. Safety pharmacology studies for human pharmaceuticals S7A. Available at http://www.ich.org/ fileadmin/Public_Web_Site/ICH_Products/Guidelines/Safety/S7A/ Step4/S7A_Guideline.pdf. Accessed January 2012

13 Adeyemi OO, Akindele AJ, Yemitan OK, Aigbe FR, Fagbo FI. Anticonvulsant, anxiolytic and sedative activities of the aqueous root extract of Securidaca longepedunculata Fresen. J Ethnopharmacol 2010; 130: 191-195

14 Rodriguez-Burford C, Oelschlager DK, Talley LI, Barnes MN, Partridge EE, Grizzle WE. The use of dimethylsulfoxide as a vehicle in cell culture experiments using ovarian carcinoma cell lines. Biotech Histochem 2003; 78: $17-21$

15 Lison D, Huaux F. In vitro studies: Ups and downs of cellular uptake. Nat Nanotechnol 2011; 6: 332-333

16 Subramanian R, Asmawi MZ, Sadikun A. In vitro alpha-glucosidase and alpha-amylase enzyme inhibitory effects of Andrographis paniculata extract and andrographolide. Acta Biochim Pol 2008; 55: 391-398

17 Matsui T, Kobayashi M, Hayashida S, Matsumoto K. Luteolin, a flavone, does not suppress postprandial glucose absorption through an inhibition of alpha-glucosidase action. Biosci Biotechnol Biochem 2002; 66: 689-692

18 Palu A, Deng S, West B, Jensen J. Xanthine oxidase inhibiting effects of noni (Morinda citrifolia) fruit juice. Phytother Res 2009; 23: 17901791

19 Verpoorte R. Setting standards! J Ethnopharmacol 2006; 106: 289

20 Cos P, Vlietinck AJ, Berghe DV, Maes L. Anti-infective potential of natural products: how to develop a stronger in vitro 'proof-of-concept'. J Ethnopharmacol 2006; 106: 290-302

21 Sarawek S, Feistel B, Pischel I, Butterweck V. Flavonoids of Cynara scolymus possess potent xanthinoxidase inhibitory activity in vitro but are devoid of hypouricemic effects in rats after oral application. Planta Med 2008; 74: 221-227

22 Sarawek S, Derendorf H, Butterweck V. Pharmacokinetics of luteolin and metabolites in rats. Nat Prod Commun 2008; 3: 2029-2036

23 Reagan-Shaw S, Nihal M, Ahmad N. Dose translation from animal to human studies revisited. FASEB J 2008; 22: 659-661

24 Baur JA, Pearson KJ, Price NL, Jamieson HA, Lerin C, Kalra A, Prabhu VV, Allard JS, Lopez-Lluch G, Lewis K, Pistell PJ, Poosala S, Becker KG, Boss O,
Gwinn D, Wang M, Ramaswamy S, Fishbein KW, Spencer RG, Lakatta EG, Le Couteur D, Shaw RJ, Navas P, Puigserver P, Ingram DK, de Cabo R, Sinclair DA. Resveratrol improves health and survival of mice on a highcalorie diet. Nature 2006; 444: 337-342

25 Center for Drug Evaluation and Research, Center for Biological and Research. Estimating the safe and starting dose in clinical trials for therapeutics in adult healthy volunteers. Rockville, Maryland, USA: US Food and Drug Administration; 2002

26 Nebendahl K, Hauff P. Drug administration. In: Kiessling F, Pichler BJ Hauff P, editors. Small animal imaging. Heidelberg: Springer; 2011: 93-119

27 Svendsen 0 . Ethics and animal welfare related to in vivo pharmacology and toxicology in laboratory animals. Basic Clin Pharmacol Toxicol 2005; 97: 197-199

28 Claassen $V$. Neglected factors in pharmacology and neuroscience research. In: Huston JP, editor. Techniques in the behavioural and neural sciences. Amsterdam: Elsevier; 1994: 46-58

29 Gotloib L, Wajsbrot V, Shostak A. A short review of experimental peritoneal sclerosis: from mice to men. Int J Artif Organs 2005; 28: 97-104

30 Ryabinin AE, Wang YM, Finn DA. Different levels of Fos immunoreactivity after repeated handling and injection stress in two inbred strains of mice. Pharmacol Biochem Behav 1999; 63: 143-151

31 Wright DM, Lincoln DW. Stress-induced analgesia evoked by intraperitoneal injection of hypertonic saline: evidence for its occurrence in vasopressin deficient rats. Physiol Behav 1985; 34: 691-695

32 de-Paris F, Neves G, Salgueiro JB, Quevedo J, Izquierdo I, Rates SM. Psychopharmacological screening of Pfaffia glomerata Spreng. (Amaranthaceae) in rodents. J Ethnopharmacol 2000; 73: 261-269

33 Akao T, Yoshino T, Kobashi K, Hattori M. Evaluation of salicin as an antipyretic prodrug that does not cause gastric injury. Planta Med 2002; 68: 714-718

34 Atkinson C, Berman S, Humbert O, Lampe JW. In vitro incubation of human feces with daidzein and antibiotics suggests interindividual differences in the bacteria responsible for equol production. J Nutr 2004; 134: 596-599

35 Mullen W, Rouanet JM, Auger C, Teissedre PL, Caldwell ST, Hartley RC, Lean ME, Edwards CA, Crozier A. Bioavailability of [2-(14)C]quercetin4'-glucoside in rats. J Agric Food Chem 2008; 56: 12127-12137

36 Aura AM, O'Leary KA, Williamson G, Ojala M, Bailey M, Puupponen-Pimia R, Nuutila AM, Oksman-Caldentey KM, Poutanen K. Quercetin derivatives are deconjugated and converted to hydroxyphenylacetic acids but not methylated by human fecal flora in vitro. J Agric Food Chem 2002; 50: 1725-1730

37 Blaut M, Schoefer L, Braune A. Transformation of flavonoids by intestinal microorganisms. Int J Vitam Nutr Res 2003; 73: 79-87

38 Griffiths LA, Smith GE. Metabolism of myricetin and related compounds in the rat. Metabolite formation in vivo and by the intestinal microflora in vitro. Biochem J 1972; 130: 141-151

39 Keppler K, Hein EM, Humpf HU. Metabolism of quercetin and rutin by the pig caecal microflora prepared by freeze-preservation. Mol Nutr Food Res 2006; 50: 686-695

40 Labib S, Hummel S, Richling E, Humpf HU, Schreier P. Use of the pig caecum model to mimic the human intestinal metabolism of hispidulin and related compounds. Mol Nutr Food Res 2006; 50: 78-86

41 Scalbert A, Morand C, Manach C, Remesy C. Absorption and metabolism of polyphenols in the gut and impact on health. Biomed Pharmacother 2002; 56: 276-282

42 Vissiennon C, Nieber K, Kelber O, Butterweck V. Route of administration determines the anxiolytic activity of the flavonols kaempferol, quercetin and myricetin - are they prodrugs? J Nutr Biochem, in press

43 Butterweck V, Lieflander-Wulf U, Winterhoff H, Nahrstedt A. Plasma levels of hypericin in presence of procyanidin B2 and hyperoside: a pharmacokinetic study in rats. Planta Med 2003; 69: 189-192

44 Nahrstedt A, Butterweck $V$. Lessons learned from herbal medicinal products: the example of St. John's Wort. J Nat Prod 2010; 73: 10151021

45 Gertsch J. Botanical drugs, synergy, and network pharmacology: forth and back to intelligent mixtures. Planta Med 2011; 77: 1086-1098 